\title{
Using caputo-fabrizio derivative for the transmission of mathematical model epidemic Corona Virus
}

\author{
M. Tahir ${ }^{1}$. G. Zaman ${ }^{2}$. S. I. A Shah ${ }^{3}$
}

Received: 21 April 2020 / Accepted: 24 August 2020 / Published online: 26 September 2020

(c) The Author(s) 2020

\begin{abstract}
Just in a week a rapidly spreading corona virus which was originated in Wuhan, city of China, infected more than 20,000 people and also killed at least 427 people in that week worldwide. Corona virus is transmissible and spreading from person to person, while the Chinese commanded authorities are scrambling to treat a flood of new patients in Chines successfully. The said Corona virus has been spread from an initial outbreak in Wuhan, city of China, and invade 25 other worldwide countries. In this article, we considered the mathematical model (Chen et al. Infect Dis Poverty, https://doi.org/10.1186/s40249-020-006403 ) in which Bats-Hosts-Reservoir-People and their transmission was taken, while we introduced the population of susceptible Bats and visitors to Wuhan city or any country in same mathematical model. Now we studying two types of populations first Bats-Hosts-ReservoirPeople (Chen et al. Infect Dis Poverty, https://doi.org/10.1186/s40249-020-00640-3, also introducing susceptible Bats and second visitors to Wuhan city, china or any country in the same model. We used Caputo-Fabrizio derivative with provided result that the addition of susceptible Bats and visitors are not responsible in spread of infection. The numerical result also supported our model.
\end{abstract}

Keyword Reservoir to person population(RP), Visitors population(VP), Mathematical model, $R_{0}$, Caputo-Fabrizio derivative, Numerical Simulation.

M. Tahir

tahir@northern.edu.pk

G. Zaman

talash74@yahoo.com

S. I. A Shah

inayat64@gmail.com

1 Department Of Mathematics, Northern University, Nowshera, KPK, Pakistan

2 Department of Mathematics, University of Malakand, Chakdara District Lower Dir, KPK, Pakistan

3 Department Of Mathematics, Islamia College University, 25000 Peshawar, Pakistan 


\section{Background}

The people of the entire world seen a very new epidemic thread in end of 2019 and 2020. After the middle East Respiratory Syndrome(MERS), in middle east, a new virus Corona attacked the "Wuhan city, China". The Corona virus was first emerged in late December and has killed more than 2500 people there, this means "Wuhan", China alone accounted for nearly 80 percent of the country's with total deaths occur 3299 or more. Before this, the World Health Organization (WHO) China Country Office informed in 31, December 2019 about pneumonia(unknown cause) detected in "Wuhan" city, Hubei China and WHO announced novel Corona virus (2019-nCoV). The International Committee of Taxonomy assigned it severe acute respiratory syndrome Corona virus-2(SARS-CoV-2) on 11 February, 2020 [12, $31,34]$. Similarly some of the best approaches was studied and presented in $[4,15,18,35]$. All the country then infected, and an perception was drawn that visitors involve in this transmission of new virus mostly.

Scientists of all the world then felt an urgent mathematical model to estimate the transmission of this disease in China. Several researches focusing and developed mathematical models for MERS [21,30,32]. In these mathematical model the scientists trying the reproductive number which responsible for whole model $[10,23,32]$. The people are awarded from different methods, and control strategies to make possible precautions against Corona virus. Here the main aim is to estimate positivity, equilibrium, and Boundedness of these models $[11,13,16,27]$. The recent and new ideas was also discussed in $[1-3,5,14,19,25,26]$. While one of the best approach towards mathematical models is optimal control [17,20,22,29]. Many different methods are adopted for optimal purpose. One best attempt was done in [31].

In our work we try to vanish and prove the wrong perception that susceptible Bats and visitors are the spreading agent of Corona virus in "wuhan" or any other country. Here we focus on the study done in [9] which was Bats-Hosts-Reservoir-People(BHRP) and its different transmission ways of Corona virus in any population. We just interesting in human-to-human transmission, so for that issue we introduced Bats population and visitors population who visit Wuhan China or any other country. We developed a mathematical model for the transmission of Corona virus Reservoir-People-Visitors(RPV) with introducing susceptible bats and estimate the value of $R_{0}$. We apply Caputo-Fabrizio derivative approach towards this issue, and a numerical simulation in the last.

\section{Data source}

The named Corona virus which was COVID-19 which was SARS-CoV-2 in the first and the model was taken from the published literature [21]. The epidemic and its sharp curve was considered from 7 December, 2019 to 31 March, 2020 is collected for this study, while simulation step size is 1 week.

\section{Simulation methods and statistical analysis}

For simulation and curve fitting we have used Runge-Kutta-fourth-order method. The reproductive number for first model was considered 0.5 and also we considered $R_{0}$ is equal to 1 for Bats and visitors model. 


\section{Mathematical model and transmission of Bats-Hosts-Reservoir-People(BHRP)}

In 19 January, 2020 [8] published BHRP mathematical transmission model in bioRxiv, with the following certain assumptions:

Bats population: 1:) Divided into four section, susceptible, exposed, infected, and removed are $\left(S_{B}\right),\left(E_{B}\right),\left(I_{B}\right)$ and $\left(R_{B}\right)$ respectively. But in our model we considered susceptible Bats $\left(S_{B}\right)$ only, and ignored the remaining because of no concern in human population. While $n_{B}$ and $m_{B}$ are taken the birth and death rate. $B$ is the rate of infection from $S_{B}$ when contact with $I_{B}$.

Host Population: 2:) Population of host is also divided in four compartments: $\left(S_{H}\right),\left(E_{H}\right),\left(I_{H}\right)$ and $\left(R_{H}\right)$ denoted susceptible, exposed, infected, and removed hosts. Here we leave all the compartments of the Hosts population, due to no role in human population infection.

Reservoir Population: Here $W$ denoted SARS-CoV-2 in reservoir (the seafood market). In our study the rate of asymptomatic infected people and symptomatic infected people export virus with $\mu_{p}$ and $\hat{\mu}_{p}$ respectively from markets or any other source.

People Population: This population is divided in to five classes, there five compartments are: $\left(S_{p}\right),\left(E_{p}\right),\left(I_{p}\right),\left(A_{p}\right)$ and $\left(R_{p}\right)$ denoted susceptible, exposed, symptomatic infected, asymptomatic infected, and removed peoples compartments. Here $m_{p}$ represent death rate. $\delta_{p}$ was defined for the proportion of asymptomatic infection, also the $S_{p}$ infected from $W$ and $I_{p}$ with the transmission rate $\beta_{W}$ and $\beta_{p}$.

\section{The new bats and visitors model}

Here we drawn some more assumptions for our model:

Assumption 1 In our study we considered the Bats population only.

Assumption 2 We ignored the transmission of Bats-Host population due to unknown spreading source.

Assumption 3 Hosts population of Bats is totally ignored in this study because of its unknown relation and infection.

Assumption 4 We leave other related terms and contact in reservoir population in this article.

Assumption 5 We taken the class of susceptible Bats to check weather this class spread any infection in the concern model.

Assumption 6 In this study we introduced visitors population compartment separately.

During in any outbreak the death rate is normally high, therefore, the model BHRP converted into BRP and then in BRV(Bats-reservoir-visitors) model by the following,

$$
\begin{aligned}
S_{B}^{\bullet} & =A_{B}-m_{B} S_{B}-\beta_{B} S_{B} I_{B}, \\
S_{p}^{\bullet} & =A_{B}-m_{B} S_{B}-\beta_{B} S_{B}\left(I_{P}+\kappa A_{p}\right)-\beta_{W} S_{p} W, \\
E_{p}^{\bullet} & =\beta_{B} S_{B}\left(I_{P}+\kappa A_{p}\right)+\beta_{W} S_{p} W-\left(1-\delta_{p}\right) W_{p} E_{p}-\delta_{p} W_{p} E_{p}-m_{p} E_{p}, \\
I_{p}^{\bullet} & =\left(1-\delta_{p}\right) W_{p} E_{p}-\left(\gamma_{p}+m_{p}\right) I_{p}, \\
A_{p}^{\bullet} & =\delta_{p} W_{p}^{\prime} E_{p}-\left(\gamma_{p}^{\prime}+m_{p}\right) A_{p},
\end{aligned}
$$




$$
\begin{aligned}
R_{p}^{\bullet} & =\gamma_{p} I_{p}+\gamma_{p}^{\prime} A_{p}-m_{p} R_{p}, \\
W^{\bullet} & =\mu_{p} I_{p}+\mu_{p}^{\prime} A_{p}-\epsilon W .
\end{aligned}
$$

In outbreak the visitors and hosts interaction was very slow in "Wuhan" or other country. Now introducing dynamic population of the visitors to any city or country is as in separate for estimation of infection,

$$
\begin{aligned}
S^{\bullet} & =\mu_{N}-\sigma(t) S I-\alpha S, \\
E^{\bullet} & =\sigma(t) S I-\lambda E-\alpha E, \\
I^{\bullet} & =\lambda E-\eta I-\alpha I .
\end{aligned}
$$

\section{Transmissibility of Corona Virus or SARS-CoV-2 based on the BRP And Bats-visitors model}

In article, $R_{0}$ is assessed for transmissibility of Corona virus(SARS-CoV-2). While the, $R_{0}$ we defined as, the expected number of secondary infections with introducing any single infected individual to susceptible population $[7,29,36]$. The value of $R_{0}$ having two faces that is, $R_{0}>1$, or $R_{0}<1$, showing different characteristics of the model in any outbreak to control or for no out control. In this study, $R_{0}$ was deduced from the BRP model and Bats-visitors population by next generation matrix approach [12].

\section{Some results}

Here in this subsection of study, we given some basic definition of the fractional calculus, which we used later,

Definition A Let $H \varepsilon G^{1}(c, d)$ and $d$ is greater then $c$, also $\tau \varepsilon[0,1]$ then CFF derivative [6] is given as,

$$
P_{t}^{\tau}(h(t))=\frac{M(\tau)}{(1-\tau)} \int_{0}^{\tau} \hat{h}(x) e^{-\tau\left(\frac{\tau-x}{1-\tau}\right)} d x .
$$

Here $M(\tau)$, which implies normality with $M(0)=M(1)=1$ [6]. But if $H$ is not contain in $G^{1}(c, d)$ then we get,

$$
P_{t}^{\tau}(h(t))=\frac{\tau M(\tau)}{(1-\tau)} \int_{a}^{\tau}(h(t)-h(x)) e^{-\tau\left(\frac{\tau-x}{1-\tau}\right)} d x .
$$

Definition B When $v=\frac{(1-\tau)}{\tau} \varepsilon[0, \infty)$ and $\tau=\frac{1}{1+v} \varepsilon[0,1]$ we have the following result,

$$
P_{t}^{\tau}(h(t))=\frac{K(v)}{v} \int_{a}^{t} \hat{h}(x) e^{\left(-\frac{t-x}{v}\right)} d x .
$$

With

$$
K(0)=K(\infty)=1
$$

Applying $\lim v \rightarrow 0$ we get,

$$
P_{t}^{\tau}(h(t))=\lim _{v \rightarrow 0} \frac{1}{v} e^{\left(-\frac{t-x}{v}\right)} d x=v(x-t) .
$$

This integral definition was provided by Losada and J. Nieto [24]. 
Definition C Suppose that " $0<\tau<1$ " then CFFD integral of given function $h$ is as,

$$
I_{t}^{\tau} h(t)=\frac{2(1-\tau)}{(2-\tau) M(\tau)} h(\tau)+\frac{2 \tau}{(2-\tau) M(\tau))} \int_{0}^{t} h(s) d s, t \geq 0 .
$$

The above equation will also be written in the following as,

$$
\frac{2(1-\tau)}{(2-\tau) M(\tau)}+\frac{2 \tau}{(2-\tau) M(\tau))}=1
$$

This gives us that $M(\tau)=\frac{2}{(2-\tau)}$, with $0<\tau<1$. From Eq. (3) a new form of the above "Caputo-Fabrizio fractinal derivative of order $0<\tau<1$ which was further investigated by Losada and J. Nieto [24].

$$
P_{t}^{\tau} h(t)=\frac{1}{(1-\tau)} \int_{0}^{t} \hat{h}(x) e^{\left(\tau\left(\frac{(t-x)}{(1-\tau)}\right)\right.} d x
$$

We use the above form in mathematical model of HBV and diabetes model $[28,33]$.

\section{Formulation of Model BVP from BRPV}

Now we are going to replace system (1) by the new CF fractional derivative, as,

$$
\begin{aligned}
C_{0} F D_{t}^{\tau} S_{B} & =A_{B}-m_{B} S_{B}-\beta_{B} S_{B} I_{B}, \\
C_{0} F D_{t}^{\tau} S_{p} & =A_{B}-m_{B} S_{B}-\beta_{B} S_{B}\left(I_{P}+\kappa A_{p}\right)-\beta_{W} S_{p} W, \\
C_{0} F D_{t}^{\tau} E_{p} & =\beta_{B} S_{B}\left(I_{P}+\kappa A_{p}\right)+\beta_{W} S_{p} W-\left(1-\delta_{p}\right) W_{p} E_{p}-\delta_{p} W_{p} E_{p}-m_{p} E_{p}, \\
C_{0} F D_{t}^{\tau} I_{p} & =\left(1-\delta_{p}\right) W_{p} E_{p}-\left(\gamma_{p}+m_{p}\right) I_{p}, \\
C_{0} F D_{t}^{\tau} A_{p} & =\delta_{p} W_{p}^{\prime} E_{p}-\left(\gamma_{p}^{\prime}+m_{p}\right) A_{p}, \\
C_{0} F D_{t}^{\tau} R_{p} & =\gamma_{p} I_{p}+\gamma_{p}^{\prime} A_{p}-m_{p} R_{p}, \\
C_{0} F D_{t}^{\tau} W & =\mu_{p} I_{p}+\mu_{p}^{\prime} A_{p}-\epsilon W .
\end{aligned}
$$

Similarly we also replace Eq. (2) by CF fractional derivative by below,

$$
\begin{aligned}
& C_{0} F D_{t}^{\tau} S=\mu_{N}-\sigma(t) S I-\alpha S, \\
& C_{0} F D_{t}^{\tau} E=\sigma(t) S I-\lambda E-\alpha E, \\
& C_{0} F D_{t}^{\tau} E=\lambda E-\eta I-\alpha I .
\end{aligned}
$$

\section{Basic reproductive number}

The calculate the basic reproductive number for system (1) is as,

$$
R_{0}=\frac{A_{B}\left(1-\delta_{p}\right) W_{p}+\left(\gamma_{p} I_{p}\right) A_{p}}{\mu_{p}+\mu_{p}^{\prime} A_{p}-W}+\frac{\beta_{W} A_{p}}{\left(\gamma_{p}^{\prime}+m_{p}\right)} .
$$




\section{Methodology for our model}

For existence for the model we use Fixed point theory. Now Caputo-Fabrizio fractional derivative for system (1), is,

$$
\begin{aligned}
S_{B}(t)-S_{B}(0)= & C_{0} F I_{t}^{\tau}\left\{A_{B}-m_{B} S_{B}-\beta_{B} S_{B} I_{B}\right\}, \\
S_{p}(t)-S_{p}(0)= & C_{0} F I_{t}^{\tau}\left\{A_{B}-m_{B} S_{B}-\beta_{B} S_{B}\left(I_{P}+\kappa A_{p}\right)-\beta_{W} S_{p} W\right\}, \\
E_{p}(t)-E_{p}(0)= & C_{0} F I_{t}^{\tau}\left\{\beta_{B} S_{B}\left(I_{P}+\kappa A_{p}\right)\right. \\
& \left.+\beta_{W} S_{p} W-\left(1-\delta_{p}\right) W_{p} E_{p}-\delta_{p} W_{p} E_{p}-m_{p} E_{p}\right\}, \\
I_{p}(t)-I_{p}(0)= & C_{0} F I_{t}^{\tau}\left\{\left(1-\delta_{p}\right) W_{p} E_{p}-\left(\gamma_{p}+m_{p}\right) I_{p}\right\}, \\
A_{p}(t)-A_{p}(0)= & C_{0} F I_{t}^{\tau}\left\{\delta_{p} W_{p}^{\prime} E_{p}-\left(\gamma_{p}^{\prime}+m_{p}\right) A_{p}\right\}, \\
R_{p}(t)-R_{p}(0)= & C_{0} F I_{t}^{\tau}\left\{\gamma_{p} I_{p}+\gamma_{p}^{\prime} A_{p}-m_{p} R_{p}\right\}, \\
W(t)-W(0)= & C_{0} F I_{t}^{\tau}\left\{\mu_{p} I_{p}+\mu_{p}^{\prime} A_{p}-\epsilon W\right\} .
\end{aligned}
$$

Similarly using Caputo-Fabrizio fractional derivative for Eq. (4), is in below,

$$
\begin{aligned}
S_{(}(t)-S_{(}(0) & =C_{0} F I_{t}^{\tau}\left\{\mu_{N}-\sigma(t) S I-\alpha S\right\}, \\
E_{(}(t)-E_{(}(0) & =C_{0} F I_{t}^{\tau}\{\sigma(t) S I-\lambda E-\alpha E\}, \\
I_{(}(t)-I_{(}(0) & =C_{0} F I_{t}^{\tau}\{\lambda E-\eta I-\alpha I\} .
\end{aligned}
$$

Now we applying the idea which used in [24] on Eqs. (6), and (7), while we get Eqs. (8) and (9), from Eqs. (6), and (7),

$$
\begin{aligned}
S_{B}(t)-S_{B}(0)= & \frac{2(1-\tau)}{(2-\tau) M(\tau)}\left\{A_{B}-m_{B} S_{B}-\beta_{B} S_{B} I_{B}\right\} \\
+ & \frac{2 \tau}{(2-\tau) M(\tau)} \int_{0}^{t}\left\{A_{B}-m_{B} S_{B}-\beta_{B} S_{B} I_{B}\right\} d y \\
S_{p}(t)-S_{p}(0)= & \frac{2(1-\tau)}{(2-\tau) M(\tau)}\left\{A_{B}-m_{B} S_{B}-\beta_{B} S_{B}\left(I_{P}+\kappa A_{p}\right)-\beta_{W} S_{p} W\right\} \int_{0}^{t}\left\{A_{B}-m_{B} S_{B}\right. \\
& \left.-\beta_{B} S_{B}\left(I_{P}+\kappa A_{p}\right)-\beta_{W} S_{p} W\right\} d y, \\
E_{p}(t)-E_{p}(0)= & \frac{2(1-\tau)}{(2-\tau) M(\tau)}\left\{\beta_{B} S_{B}\left(I_{P}+\kappa A_{p}\right)+\beta_{W} S_{p} W\right. \\
& \left.-\left(1-\delta_{p}\right) W_{p} E_{p}-\delta_{p} W_{p} E_{p}-m_{p} E_{p}\right\} \int_{0}^{t}\left\{A_{B}-m_{B} S_{B}\right. \\
& \left.-\beta_{B} S_{B}\left(I_{P}+\kappa A_{p}\right)+\beta_{W} S_{p} W-\left(1-\delta_{p}\right) W_{p} E_{p}-\delta_{p} W_{p} E_{p}-m_{p} E_{p}\right\} d y, \\
I_{p}(t)-I_{p}(0)= & \left.\frac{2(1-\tau)}{(2-\tau) M(\tau)}\left\{1-\delta_{p}\right) W_{p} E_{p}-\left(\gamma_{p}+m_{p}\right) I_{p}\right\} \\
& \left.+\frac{2 \tau}{(2-\tau) M(\tau)} \int_{0}^{t}\left\{1-\delta_{p}\right) W_{p} E_{p}-\left(\gamma_{p}+m_{p}\right) I_{p}\right\} d y, \\
& \frac{2(1-\tau)}{(2-\tau) M(\tau)}\left\{\delta_{p} W_{p}^{\prime} E_{p}-\left(\gamma_{p}^{\prime}+m_{p}\right) A_{p}\right\} \\
& +\frac{2 \tau}{(2-\tau) M(\tau)} \int_{0}^{t}\left\{\delta_{p} W_{p}^{\prime} E_{p}-\left(\gamma_{p}^{\prime}+m_{p}\right) A_{p}\right\} d y, \\
A_{p}(t)-A_{p}(0)= & \frac{2(1-\tau)}{(2-\tau) M(\tau)}\left\{\gamma_{p} I_{p}+\gamma_{p}^{\prime} A_{p}-m_{p} R_{p}\right\} \\
& +\frac{2 \tau}{(2-\tau) M(\tau)} \int_{0}^{t}\left\{\gamma_{p} I_{p}+\gamma_{p}^{\prime} A_{p}-m_{p} R_{p}\right\} d y, \\
R_{p}(t)-R_{p}(0)= &
\end{aligned}
$$




$$
\begin{aligned}
W(t)-W(0)= & \frac{2(1-\tau)}{(2-\tau) M(\tau)}\left\{\mu_{p} I_{p}+\mu_{p}^{\prime} A_{p}-\epsilon W\right\} \\
& \quad+\frac{2 \tau}{(2-\tau) M(\tau)} \int_{0}^{t}\left\{\mu_{p} I_{p}+\mu_{p}^{\prime} A_{p}-\epsilon W\right\} d y .
\end{aligned}
$$

Here we applying the new idea of Losada and J.Nieto [24] on the system given in above, and we get,

$$
\begin{aligned}
S_{(}(t)-S_{(}(0)= & \frac{2(1-\tau)}{(2-\tau) M(\tau)}\left\{\mu_{N}-\sigma(t) S I-\alpha S\right\}+\frac{2 \tau}{(2-\tau) M(\tau)} \int_{0}^{t}\left\{\mu_{N}-\sigma(t) S I-\alpha S\right\}, \\
E_{(}(t)-E_{(}(0)= & \frac{2(1-\tau)}{(2-\tau) M(\tau)}\{\sigma(t) S I-\lambda E-\alpha E\} \\
& +\frac{2 \tau}{(2-\tau) M(\tau)} \int_{0}^{t}\{\sigma(t) S I-\lambda E-\alpha E\} d y, \\
S_{B}(t)-S_{B}(0)= & \frac{2(1-\tau)}{(2-\tau) M(\tau)}\{\lambda E-\eta I-\alpha I\} \\
& +\frac{2 \tau}{(2-\tau) M(\tau)} \int_{0}^{t}\{\lambda E-\eta I-\alpha I\} d y .
\end{aligned}
$$

Now the simplified form of the Eqs. (10) and (11), after Eqs. (8) and (9), is below,

$$
\begin{aligned}
\Phi_{1}\left(t, S_{B}\right) & =\left\{A_{B}-m_{B} S_{B}-\beta_{B} S_{B} I_{B}\right\} \\
\Phi_{2}\left(t, S_{p}\right) & =\left\{A_{B}-m_{B} S_{B}-\beta_{B} S_{B}\left(I_{P}+\kappa A_{p}\right)-\beta_{W} S_{p} W\right\} \\
\Phi_{3}\left(t, E_{p}\right) & =\left\{\beta_{B} S_{B}\left(I_{P}+\kappa A_{p}\right)+\beta_{W} S_{p} W-\left(1-\delta_{p}\right) W_{p} E_{p}-\delta_{p} W_{p} E_{p}-m_{p} E_{p}\right\} \\
\Phi_{4}\left(t, I_{p}\right) & =\left\{\left(1-\delta_{p}\right) W_{p} E_{p}-\left(\gamma_{p}+m_{p}\right) I_{p}\right\} \\
\Phi_{5}\left(t, A_{p}\right) & =\left\{\delta_{p} W_{p}^{\prime} E_{p}-\left(\gamma_{p}^{\prime}+m_{p}\right) A_{p}\right\} \\
\Phi_{6}\left(t, R_{p}\right) & =\left\{\gamma_{p} I_{p}+\gamma_{p}^{\prime} A_{p}-m_{p} R_{p}\right\} \\
\Phi_{7}(t, W) & =\left\{\mu_{p} I_{p}+\mu_{p}^{\prime} A_{p}-\epsilon W\right\} \\
\prod_{1}(t, S) & =\left\{\mu_{N}-\sigma(t) S I-\alpha S\right\} \\
\prod_{2}(t, S) & =\{\sigma(t) S I-\lambda E-\alpha E\} \\
\prod_{3}(t, S) & =\{\lambda E-\eta I-\alpha I\} .
\end{aligned}
$$

Theorem 10.1 The Kernals of $\Phi_{1}, \Phi_{2}, \Phi_{3}, \Phi_{4}, \Phi_{5}, \Phi_{6}$ and $\Phi_{7}$ fulfill the Lipschitz condition and contraction if the following inequality hold. $0 \leq\left(m_{B}+\beta_{m} \psi\right) e+I_{B}<1$, where $\psi=1$ and $e=\frac{1}{2}$.

Proof We prove the theorem for $\Phi_{1}, \Phi_{2}, \Phi_{3}, \Phi_{4}, \Phi_{5}, \Phi_{6}$ and $\Phi_{7}$ respectively. First suppose that $S$ and $S_{1}$ are two function for $\Phi_{1}$, then,

$$
\left\|\Phi_{1}\left(t, S_{B}\right)-\Phi_{1}\left(t, S_{1 B}\right)\right\|=\left\|-m_{B} S_{B}\left\{S_{B}(t)-S_{B}\left(t_{1}\right)\right\}-\beta_{B} I_{B}\left\{S_{B}(t)-S_{B}\left(t_{1}\right)\right\}\right\| .
$$

For Eq. (12) we apply triangle in-equality, we get,

$$
\begin{aligned}
\| \Phi_{1}\left(t, S_{B}\right) & -\Phi_{1}\left(t, S_{1 B}\right)\|\leq\|-m_{B} S_{B}\left\{S_{B}(t)-S_{B}\left(t_{1}\right)\right\} \| \\
& +\left\|\left\{-\beta_{B} I_{B}\left\{S_{B}(t)-S_{B}\left(t_{1}\right)\right\}\right\}\right\| . \\
& \leq\left\|m_{B}\right\|+\|\left\{\beta_{B}\|\| I_{B} \|\left\{\left\|S_{B}(t)-S_{B}\left(t_{1}\right)\right\|\right\} .\right.
\end{aligned}
$$




$$
\begin{aligned}
& \leq\left(m_{B}+\beta_{m} \cdot 1\right)\left\{\frac{1}{2}\right\}+I_{B}\left\|S_{B}(t)-S_{B}\left(t_{1}\right)\right\| . \\
& \leq \varphi\left\|S_{B}(t)-S_{B}\left(t_{1}\right)\right\| .
\end{aligned}
$$

Here while we use $\varphi=\left(m_{B}+\beta_{m}\right)$ with $\psi=1$ and $e=\frac{1}{2}$.

This implies the given function is a bounded function, so we have,

$$
\left\|\Phi_{1}\left(t, S_{B}\right)-\Phi_{1}\left(t, S_{1 B}\right)\right\| \leq \varphi\left\|S_{B}(t)-S_{B}\left(t_{1}\right)\right\| .
$$

Hence, we see that the Lipschitz condition for Eq. (12) is satisfied, also $0 \leq\left(m_{B}+\beta_{m} \psi\right) e+$ $I_{B}<1$, where $\psi=1$ and $e=\frac{1}{2}$, which also emphasized that the said Eq. (12) is contraction. The Lipschitz condition for other equations by the similar way are given below,

$$
\begin{gathered}
\left\|\Phi_{2}\left(t, S_{p}\right)-\Phi_{2}\left(t, S_{1 p}\right)\right\| \leq \varphi\left\|S_{p}(t)-S_{p}\left(t_{1}\right)\right\| . \\
\left\|\Phi_{3}\left(t, E_{p}\right)-\Phi_{2}\left(t, E_{1 p}\right)\right\| \leq \varphi_{1}\left\|E_{p}(t)-E_{p}\left(t_{1}\right)\right\| . \\
\left\|\Phi_{4}\left(t, I_{p}\right)-\Phi_{2}\left(t, I_{1 p}\right)\right\| \leq \varphi_{2}\left\|I_{p}(t)-I_{p}\left(t_{1}\right)\right\| . \\
\left\|\Phi_{5}-\Phi_{2}\left(t, A_{1 p}\right)\right\| \leq \varphi_{3}\left\|A_{p}(t)-A_{p}\left(t_{1}\right)\right\| . \\
\left\|\Phi_{6}\left(t, R_{p}\right)-\Phi_{2}\left(t, R_{1 p}\right)\right\| \leq \varphi_{4}\left\|R_{p}(t)-R_{p}\left(t_{1}\right)\right\| . \\
\left\|\Phi_{7}(t, W)-\Phi_{2}(t, W)\right\| \leq \varphi_{5}\left\|W(t)-W\left(t_{1}\right)\right\| .
\end{gathered}
$$

Theorem 10.2 The Kernals of Eq. (10) $\prod_{1}, \prod_{2}$ and $\prod_{3}$ fulfill the Lipschitz, condition and contraction if the following inequality hold. $0 \leq(\sigma(t)+\alpha \psi) e+I<1$, where $\psi=1$ and $I=e=\frac{1}{2}$.

Proof To prove the concern condition suppose that $D$ and $D_{1}$ are any two function then, from $\prod_{1}$ we write as,

$$
\left\|\prod_{1}(t, D)-\prod_{1}\left(t, D_{1}\right)\right\|=\|-\sigma(t) S I-\alpha S\| .
$$

By triangle inequality Eq. (15) becomes,

$$
\begin{aligned}
\left\|\prod_{1}(t, D)-\prod_{1}\left(t, D_{1}\right)\right\| & \leq\|-\sigma(t) S I\|+\|-\alpha S\| . \\
& \leq\|\sigma(t) S I\|+\|\alpha S\| . \\
& \leq\left\|\sigma(t) I S\left\{D(t)-D_{1}(t)\right\}\right\|+\left\|\alpha S\left\{D(t)-D_{1}(t)\right\}\right\| . \\
& \leq\|\sigma(t)\|\|I\|+\|\alpha\|\left\|\left\{D(t)-D_{1}(t)\right\}\right\| . \\
& \leq\{\sigma(t)+\alpha \cdot 1\}\left\{\frac{1}{2}\right\} \|\left\{D(t)-D_{1}(t) \|\right\} . \\
& \leq \Omega \|\left\{D(t)-D_{1}(t) \| .\right.
\end{aligned}
$$

Where $\Omega=\sigma(t)+\alpha$, while again we see that $\psi=1$ and $I=e=\frac{1}{2}$ showing the same effect of disease spread rate through visitors to "Wahan" city or the people living there in "Wahan".

Hence, Lipschitz condition for Eq. (16) is satisfied, and by similar way we find the remaining Eq. (10) are below, as,

$$
\begin{aligned}
& \left.\left.\left\|\prod_{1}(t, D)-\prod_{1}\left(t, D_{1}\right)\right\| \leq \Omega_{1} \| E_{(} t\right)-E_{(} t_{1}\right) \| . \\
& \left.\left\|\prod_{2}(t, D)-\prod_{2}\left(t, D_{1}\right)\right\| \leq \Omega_{2} \| I_{(} t\right)-I_{(}\left(t_{1}\right) \| .
\end{aligned}
$$


Conclusion : 1 In this subsection we conclude that both the population showing the same effect on the new disease "Corona Virus" spread in China "Wahan" city. The Eqs. (13), (14), (16), and (17) providing the same values i.e, $\psi=1$ and $e=\frac{1}{2}$, indicated that if the visitors visit the particular city or no visitors there in the concern city the spreading ratio of the disease remain same from the fixed values taken both population. These fixed values of both the population $\psi$ and $e$ create a perception that visitors have not a key role in this epidemic disease which spread in particular region of the "Wahan" city.

Taking Eqs. (8) and (9) with kernal notation becomes,

$$
\begin{aligned}
& S_{B}(t)=S_{B}(0)+\frac{2(1-\tau)}{(2-\tau) M(\tau)}\left\{\Phi_{1}\left(t, S_{B}\right)\right\}+\frac{2 \tau}{(2-\tau) M(\tau)} \int_{0}^{t}\left\{\Phi_{1}\left(y, S_{B}\right)\right\} d y, \\
& S_{p}(t)=S_{p}(0)+\frac{2(1-\tau)}{(2-\tau) M(\tau)}\left\{\Phi_{2}\left(t, S_{p}\right)\right\}+\frac{2 \tau}{(2-\tau) M(\tau)} \int_{0}^{t}\left\{\Phi_{2}\left(y, S_{p}\right)\right\} d y, \\
& E_{p}(t)=E_{p}(0)+\frac{2(1-\tau)}{(2-\tau) M(\tau)}\left\{\Phi_{3}\left(t, E_{p}\right)\right\}+\frac{2 \tau}{(2-\tau) M(\tau)} \int_{0}^{t}\left\{\Phi_{3}\left(y, E_{p}\right)\right\} d y, \\
& I_{p}(t)=I_{p}(0)+\frac{2(1-\tau)}{(2-\tau) M(\tau)}\left\{\Phi_{4}\left(t, I_{p}\right)\right\}+\frac{2 \tau}{(2-\tau) M(\tau)} \int_{0}^{t}\left\{\Phi_{4}\left(y, I_{p}\right)\right\} d y, \\
& A_{p}(t)=A_{p}(0)+\frac{2(1-\tau)}{(2-\tau) M(\tau)}\left\{\Phi_{5}\left(t, A_{p}\right)\right\}+\frac{2 \tau}{(2-\tau) M(\tau)} \int_{0}^{t}\left\{\Phi_{5}\left(y, A_{p}\right)\right\} d y, \\
& R_{p}(t)=R_{p}(0)+\frac{2(1-\tau)}{(2-\tau) M(\tau)}\left\{\Phi_{6}\left(t, R_{p}\right)\right\}+\frac{2 \tau}{(2-\tau) M(\tau)} \int_{0}^{t}\left\{\Phi_{6}\left(y, R_{p}\right)\right\} d y, \\
& W(t)=W(0)+\frac{2(1-\tau)}{(2-\tau) M(\tau)}\left\{\Phi_{7}(t, W \sigma)\right\}+\frac{2 \tau}{(2-\tau) M(\tau)} \int_{0}^{t}\left\{\Phi_{7}(y, W)\right\} d y .
\end{aligned}
$$

Using technique in Eq. (19), we obtain Eqs. (17) and (18) as Eqs.(20) and (21),

$$
\begin{aligned}
& S(t)=S(0)+\frac{2(1-\tau)}{(2-\tau) M(\tau)}\left\{\prod_{1}(t, S)\right\}+\frac{2}{(2-\tau) M(\tau)} \int_{0}^{t}\left\{\prod_{1}(y, S)\right\} d y, \\
& E(t)=E(0)+\frac{2(1-\tau)}{(2-\tau) M(\tau)}\left\{\prod_{2}(t, E)\right\}+\frac{2}{(2-\tau) M(\tau)} \int_{0}^{t}\left\{\prod_{1}(y, E)\right\} d y, \\
& I(t)=I(0)+\frac{2(1-\tau)}{(2-\tau) M(\tau)}\left\{\prod_{3}(t, I)\right\}+\frac{2}{(2-\tau) M(\tau)} \int_{0}^{t}\left\{\prod_{1}(y, I)\right\} d y .
\end{aligned}
$$

Using recurrence formula for Eqs. (19) and (20) we get,

$$
\begin{aligned}
S_{B n}(t) & =\frac{2(1-\tau)}{(2-\tau) M(\tau)}\left\{\Phi_{1}\left(t, S_{B}(n-1)\right)\right\}+\frac{2 \tau}{(2-\tau) M(\tau)} \int_{0}^{t}\left\{\Phi_{1}\left(y, S_{B}(n-1)\right)\right\} d y, \\
S_{p n}(t) & =\frac{2(1-\tau)}{(2-\tau) M(\tau)}\left\{\Phi_{2}\left(t, S_{p}(n-1)\right)\right\}+\frac{2 \tau}{(2-\tau) M(\tau)} \int_{0}^{t}\left\{\Phi_{1}\left(y, S_{p}(n-1)\right)\right\} d y, \\
E_{p n}(t) & =\frac{2(1-\tau)}{(2-\tau) M(\tau)}\left\{\Phi_{3}\left(t, E_{p}(n-1)\right)\right\}+\frac{2 \tau}{(2-\tau) M(\tau)} \int_{0}^{t}\left\{\Phi_{1}\left(y, E_{p}(n-1)\right)\right\} d, \\
I_{p n}(t) & =\frac{2(1-\tau)}{(2-\tau) M(\tau)}\left\{\Phi_{4}\left(t, I_{p}(n-1)\right)\right\}+\frac{2 \tau}{(2-\tau) M(\tau)} \int_{0}^{t}\left\{\Phi_{4}\left(y, I_{p}(n-1)\right)\right\} d y, \\
A_{p n}(t) & =\frac{2(1-\tau)}{(2-\tau) M(\tau)}\left\{\Phi_{5}\left(t, A_{p}(n-1)\right)\right\}+\frac{2 \tau}{(2-\tau) M(\tau)} \int_{0}^{t}\left\{\Phi_{5}\left(y, A_{p}(n-1)\right)\right\} d y,
\end{aligned}
$$




$$
\begin{aligned}
R_{p n}(t) & =\frac{2(1-\tau)}{(2-\tau) M(\tau)}\left\{\Phi_{6}\left(t, R_{p}(n-1)\right)\right\}+\frac{2 \tau}{(2-\tau) M(\tau)} \int_{0}^{t}\left\{\Phi_{6}\left(y, R_{p}(n-1)\right)\right\} d y, \\
W_{n}(t) & =\frac{2(1-\tau)}{(2-\tau) M(\tau)}\left\{\Phi_{7}(t, W(n-1))\right\}+\frac{2 \tau}{(2-\tau) M(\tau)} \int_{0}^{t}\left\{\Phi_{7}(y, W(n-1))\right\} d y(21)
\end{aligned}
$$

And we get also

$$
\begin{aligned}
S_{n}(t) & =\frac{2(1-\tau)}{(2-\tau) M(\tau)}\left\{\prod_{1}(t, S(n-1))\right\}+\frac{2}{(2-\tau) M(\tau)} \int_{0}^{t}\left\{\prod_{1}(y, S(n-1))\right\} d y, \\
E_{n}(t) & =\frac{2(1-\tau)}{(2-\tau) M(\tau)}\left\{\prod_{2}(t, E(n-1))\right\}+\frac{2}{(2-\tau) M(\tau)} \int_{0}^{t}\left\{\prod_{1}(y, E(n-1))\right\} d y, \\
I_{n}(t) & =\frac{2(1-\tau)}{(2-\tau) M(\tau)}\left\{\prod_{3}(t, I(n-1))\right\}+\frac{2}{(2-\tau) M(\tau)} \int_{0}^{t}\left\{\prod_{3}(y, I(n-1))\right\} d y .
\end{aligned}
$$

With the initial conditions for Eqs. (21) and (22) are,

$S_{B}^{0}(t)=S_{B}(t), S_{p}^{0}(t)=S_{p}(t), E_{p}^{0}(t)=E_{p}(t), I_{p}^{0}(t)=I_{p}(t), A_{p}^{0}(t)=A_{p}(t), R_{p}^{0}(t)=R_{p}(t), W^{0}(t)=W(t)$.

Now to calculate the successive terms, we use the following difference formula,

$$
\begin{aligned}
\Gamma_{1} n(t)= & S_{B} n(t)-S_{B}(n-1) t \\
= & \frac{2(1-\tau)}{(2-\tau) M(\tau)}\left\{\Phi_{1}\left(t, S_{B}(n-1)-\phi_{1}\left(t, S_{B}(n-2)\right)\right\}\right. \\
& +\frac{2 \tau}{(2-\tau) M(\tau)} \int_{0}^{t}\left\{\Phi_{1}\left(y, S_{B}(n-1)-\phi_{1}\left(y, S_{B}(n-2)\right)\right\} d y,\right. \\
\Gamma_{2} n(t)=S_{p} n(t)-S_{p}(n-1) t= & \frac{2(1-\tau)}{(2-\tau) M(\tau)}\left\{\Phi_{2}\left(t, S_{p}(n-1)-\phi_{2}\left(t, S_{p}(n-2)\right)\right\}\right. \\
& +\frac{2 \tau}{(2-\tau) M(\tau)} \int_{0}^{t}\left\{\Phi_{2}\left(y, S_{p}(n-1)-\phi_{2}\left(y, S_{p}(n-2)\right)\right\} d y,\right. \\
\Gamma_{3} n(t)=E_{p} n(t)-E_{p}(n-1) t= & \frac{2(1-\tau)}{(2-\tau) M(\tau)}\left\{\Phi_{3}\left(t, E_{p}(n-1)-\phi_{3}\left(t, E_{p}(n-2)\right)\right\}\right. \\
& +\frac{2 \tau}{(2-\tau) M(\tau)} \int_{0}^{t}\left\{\Phi_{3}\left(y, E_{p}(n-1)-\phi_{3}\left(y, e_{p}(n-2)\right)\right\} d y,\right. \\
\Gamma_{4} n(t)=I_{p} n(t)-I_{p}(n-1) t= & \frac{2(1-\tau)}{(2-\tau) M(\tau)}\left\{\Phi_{4}\left(t, I_{p}(n-1)-\phi_{4}\left(t, I_{p}(n-2)\right)\right\}\right. \\
& +\frac{2 \tau}{(2-\tau) M(\tau)} \int_{0}^{t}\left\{\Phi_{2}\left(y, I_{p}(n-1)-\phi_{4}\left(y, I_{p}(n-2)\right)\right\} d y,\right. \\
\Gamma_{5} n(t)=A_{p} n(t)-A_{p}(n-1) t= & \frac{2(1-\tau)}{(2-\tau) M(\tau)}\left\{\Phi_{5}\left(t, A_{p}(n-1)-\phi_{5}\left(t, A_{p}(n-2)\right)\right\}\right. \\
& +\frac{2 \tau}{(2-\tau) M(\tau)} \int_{0}^{t}\left\{\Phi_{5}\left(y, A_{p}(n-1)-\phi_{5}\left(y, A_{p}(n-2)\right)\right\} d y,\right. \\
\Gamma_{7} n(t)=W n(t)-W(n-1) t= & \frac{2(1-\tau)}{(2-\tau) M(\tau)}\left\{\Phi_{7}\left(t, W(n-1)-\phi_{7}(t, W(n-2))\right\}\right. \\
& +\frac{2 \tau}{(2-\tau) M(\tau)} \int_{0}^{t}\left\{\Phi_{7}\left(y, W(n-1)-\phi_{7}(y, W(n-2))\right\} d y .\right. \\
\Gamma_{6} n(t)=R_{p} n(t)-R_{p}(n-1) t= & \frac{2(1-\tau)}{(2-\tau) M(\tau)}\left\{\Phi_{6}\left(t, R_{p}(n-1)-\phi_{6}\left(t, R_{p}(n-2)\right)\right\}\right. \\
& \frac{2 \tau}{(2-\tau) M(\tau)} \int_{0}^{t}\left\{\Phi_{6}\left(y, R_{p}(n-1)-\phi_{6}\left(y, R_{p}(n-2)\right)\right\} d y,\right. \\
& \\
&
\end{aligned}
$$


Now applying the recurrence formula for visitors population dynamic in Eq. (22) is given by,

$$
\begin{aligned}
\Re_{1} n(t)= & S n(t)-S(n-1) t \\
= & \frac{2(1-\tau)}{(2-\tau) M(\tau)}\left\{\prod_{1}\left(t, S(n-1)-\prod_{1}(t, S(n-2))\right\}\right. \\
& +\frac{2}{(2-\tau) M(\tau)} \int_{0}^{t}\left\{\prod_{1}\left(y, S(n-1)-\prod_{1}(y, S(n-2))\right\} d y,\right. \\
\Re_{2} n(t)=E n(t)-E(n-1) t \quad & \frac{2(1-\tau)}{(2-\tau) M(\tau)}\left\{\prod_{2}\left(t, E(n-1)-\prod_{2}(t, E(n-2))\right\}\right. \\
& +\frac{2}{(2-\tau) M(\tau)} \int_{0}^{t}\left\{\prod_{2}\left(y, E(n-1)-\prod_{2}(y, E(n-2))\right\} d y,\right. \\
\Re_{3} n(t)=\operatorname{In}(t)-I(n-1) t \quad & \frac{2(1-\tau)}{(2-\tau) M(\tau)}\left\{\prod_{3}\left(t, S(n-1)-\prod_{3}(t, I(n-2))\right\}\right. \\
& +\frac{2}{(2-\tau) M(\tau)} \int_{0}^{t}\left\{\prod_{3}\left(y, I(n-1)-\prod_{3}(y, I(n-2))\right\} d y .\right.
\end{aligned}
$$

Here we have the following Eq. (25) with $i=1,2,3,4,5,6,7$ and Eq. (26) with $j=1,2,3$ are given,

$$
\begin{aligned}
& S_{B} n(t)=\sum_{i=1}^{n} \Gamma_{1} i(t), S_{p} n(t)=\sum_{i=1}^{n} \Gamma_{2} i(t), E_{p} n(t)=\sum_{i=1}^{n} \Gamma_{3} i(t) . I_{p} n(t)=\sum_{i=1}^{n} \Gamma_{4} i(t), \\
& A_{p} n(t)=\sum_{i=1}^{n} \Gamma_{5} i(t), R_{p} n(t)=\sum_{i=1}^{n} \Gamma_{6} i(t), W n(t)=\sum_{i=1}^{n} \Gamma_{7} i(t) \\
& \operatorname{Sn}(t)=\sum_{i=1}^{n} \prod_{1} i(t), E n(t)=\sum_{i=1}^{n} \prod_{2} i(t), \\
& \operatorname{In}(t)=\sum_{i=1}^{n} \prod_{3} i(t) \text {. }
\end{aligned}
$$

Here we use the same strategy and assume the following,

$$
\begin{aligned}
\left\|\Gamma_{1} n(t)\right\|= & \left\|S_{B} n(t)-S_{B}(n-1)(t)\right\| \\
= & \| \frac{2(1-\tau)}{(2-\tau) M(\tau)}\left\{\Phi_{1}\left(t, S_{B}(n-1)-\phi_{1}\left(t, S_{B}(n-2)\right)\right\}\right. \\
& \frac{2 \tau}{(2-\tau) M(\tau)} \int_{0}^{t}\left\{\Phi_{1}\left(y, S_{B}(n-1)-\phi_{1}\left(y, S_{B}(n-2)\right)\right\} \| d y .\right.
\end{aligned}
$$

Now for above equation we using triangle inequality, we get,

$$
\begin{aligned}
\| & S_{B} n(t)-S_{B}(n-1)(t) \| \\
& \leq \| \frac{2(1-\tau)}{(2-\tau) M(\tau)}\left\{\Phi_{1}\left(t, S_{B}(n-1)-\phi_{1}\left(t, S_{B}(n-2)\right)\right\} \|\right. \\
& +\frac{2 \tau}{(2-\tau) M(\tau)} \int_{0}^{t} \|\left\{\Phi_{1}\left(y, S_{B}(n-1)-\phi_{1}\left(y, S_{B}(n-2)\right)\right\} \| d y .\right.
\end{aligned}
$$


But we have proved that the kernal satisfied the Lipschits criteria then the above becomes,

$$
\begin{aligned}
\|S n(t)-S(n-1)(t)\| \leq & \left\|\frac{2(1-\tau)}{(2-\tau) M(\tau)}\left\{\Phi_{1} \|\left\{S_{B}(n-1)-S_{B}(n-2)\right)\right\}\right\| \\
& \left.+\frac{2 \tau}{(2-\tau) M(\tau)} \Phi_{1} \int_{0}^{t} \|\left\{S_{B}(n-1)-S_{B}(n-2)\right)\right\} \| d y .
\end{aligned}
$$

From simplifying form Eq. (27) implies,

$$
\left\|\Gamma_{1} n(t)\right\| \leq \frac{2(1-\tau)}{2-\tau M(\tau)} \Phi_{1}\left\|\Gamma_{1}(n-1)(t)\right\|+\frac{2 \tau}{2-\tau(M(\tau))} \Gamma_{1} \int_{0}^{t}\left\|\Gamma_{1}(n-1) y\right\| d y .
$$

By the same way we get the following result,

$$
\begin{aligned}
\left\|\Gamma_{2} n(t)\right\| & \leq \frac{2(1-\tau)}{2-\tau M(\tau)} \Phi_{2}\left\|\Gamma_{2}(n-1)(t)\right\|+\frac{2 \tau}{2-\tau(M(\tau))} \Gamma_{2} \int_{0}^{t}\left\|\Gamma_{2}(n-1) y\right\| d y, \\
\left\|\Gamma_{3} n(t)\right\| & \leq \frac{2(1-\tau)}{2-\tau M(\tau)} \Phi_{3}\left\|\Gamma_{3}(n-1)(t)\right\|+\frac{2 \tau}{2-\tau(M(\tau))} \Gamma_{3} \int_{0}^{t}\left\|\Gamma_{3}(n-1) y\right\| d y, \\
\left\|\Gamma_{4} n(t)\right\| & \leq \frac{2(1-\tau)}{2-\tau M(\tau)} \Phi_{4}\left\|\Gamma_{4}(n-1)(t)\right\|+\frac{2 \tau}{2-\tau(M(\tau))} \Gamma_{4} \int_{0}^{t}\left\|\Gamma_{4}(n-1) y\right\| d y, \\
\left\|\Gamma_{5} n(t)\right\| & \leq \frac{2(1-\tau)}{2-\tau M(\tau)} \Phi_{5}\left\|\Gamma_{5}(n-1)(t)\right\|+\frac{2 \tau}{2-\tau(M(\tau))} \Gamma_{5} \int_{0}^{t}\left\|\Gamma_{5}(n-1) y\right\| d y, \\
\left\|\Gamma_{6} n(t)\right\| & \leq \frac{2(1-\tau)}{2-\tau M(\tau)} \Phi_{6}\left\|\Gamma_{6}(n-1)(t)\right\|+\frac{2 \tau}{2-\tau(M(\tau))} \Gamma_{6} \int_{0}^{t}\left\|\Gamma_{6}(n-1) y\right\| d y .
\end{aligned}
$$

Similarly from Eq. (24) we have below result,

$$
\begin{aligned}
& \left\|\Re_{1} n(t)\right\| \leq \frac{2(1-\tau)}{2-\tau M(\tau)} \prod_{1}\left\|\Re_{1}(n-1)(t)\right\|+\frac{2 \tau}{2-\tau(M(\tau))} \Re_{1} \int_{0}^{t}\left\|\Re_{1}(n-1) y\right\| d y, \\
& \left\|\Re_{2} n(t)\right\| \leq \frac{2(1-\tau)}{2-\tau M(\tau)} \prod_{2}\left\|\Re_{2}(n-1)(t)\right\|+\frac{2 \tau}{2-\tau(M(\tau))} \Re_{2} \int_{0}^{t}\left\|\Re_{2}(n-1) y\right\| d y, \\
& \left\|\Re_{3} n(t)\right\| \leq \frac{2(1-\tau)}{2-\tau M(\tau)} \prod_{3}\left\|\Re_{3}(n-1)(t)\right\|+\frac{2 \tau}{2-\tau(M(\tau))} \Re_{3} \int_{0}^{t}\left\|\Re_{3}(n-1) y\right\| d y .
\end{aligned}
$$

Now considered the theorem given below,

Theorem 10.3 The model defined in system (1) has exact coupled solution if the condition below hold that is we find that

$$
\frac{2(1-\tau)}{(2-\tau) M(\tau)} \Phi_{1}+\frac{2 \tau)}{(2-\tau) M(\tau)} \Phi_{1}<1
$$

Proof We shown that all the Eqs. in (28) and (29) are bounded and the functions $S_{B}, S_{p}, E_{p}, I_{p}, A_{p}, R_{p}, W$ fulfill the Lipschitz condition, so Eqs. (28) and (29) by recursive method its succeeding relation are given below, 


$$
\begin{gathered}
\left\|\Gamma_{1}\right\| \leq\left\|S_{B} n(0)\right\|\left[\frac{2(1-\tau)}{(2-\tau) M(\tau)} \Phi_{1}+\frac{2 \tau)}{(2-\tau) M(\tau)} \Phi_{1}\right]^{n}, \\
\left\|\Gamma_{2}\right\| \leq\left\|S_{p} n(0)\right\|\left[\frac{2(1-\tau)}{(2-\tau) M(\tau)} \Phi_{2}+\frac{2 \tau)}{(2-\tau) M(\tau)} \Phi_{2}\right]^{n}, \\
\left\|\Gamma_{3}\right\| \leq\left\|E_{p} n(0)\right\|\left[\frac{2(1-\tau)}{(2-\tau) M(\tau)} \Phi_{3}+\frac{2 \tau)}{(2-\tau) M(\tau)} \Phi_{3}\right]^{n}, \\
\left\|\Gamma_{4}\right\| \leq\left\|I_{p} n(0)\right\|\left[\frac{2(1-\tau)}{(2-\tau) M(\tau)} \Phi_{4}+\frac{2 \tau)}{(2-\tau) M(\tau)} \Phi_{4}\right]^{n}, \\
\left\|\Gamma_{5}\right\| \leq\left\|A_{p} n(0)\right\|\left[\frac{2(1-\tau)}{(2-\tau) M(\tau)} \Phi_{5}+\frac{2 \tau)}{(2-\tau) M(\tau)} \Phi_{5}\right]^{n}, \\
\left\|\Gamma_{6}\right\| \leq\left\|R_{p} n(0)\right\|\left[\frac{2(1-\tau)}{(2-\tau) M(\tau)} \Phi_{6}+\frac{2 \tau)}{(2-\tau) M(\tau)} \Phi_{6}\right]^{n}, \\
\left\|\Gamma_{7}\right\| \leq\|W n(0)\|\left[\frac{2(1-\tau)}{(2-\tau) M(\tau)} \Phi_{7}+\frac{2 \tau)}{(2-\tau) M(\tau)} \Phi_{7}\right]^{n} .
\end{gathered}
$$

which shows that the existence and as well as the continuity of the concern solutions is valid and proved. Furthermore, to ensure that the above function is a solution of Eq. (3), we proceed as follows:

$$
\begin{gathered}
S_{B}(t)-S_{B}(0)=S_{B} n(t)-T_{1}(t), \\
S_{p}(t)-S_{p}(0)=S_{p} n(t)-T_{2}(t), \\
E_{p}(t)-E_{p}(0)=E_{p} n(t)-T_{3}(t), \\
I_{p}(t)-I_{p}(0)=I_{p} n(t)-T_{4}(t), \\
A_{p}(t)-A_{p}(0)=A_{p} n(t)-T_{5}(t), \\
R_{p}(t)-R_{p}(0)=R_{p} n(t)-T_{6}(t), \\
W(t)-W(0)=W n(t)-T_{7}(t) .
\end{gathered}
$$

Where the terms $T_{1}(t), T_{2}(t), T_{3}(t), T_{4}(t), T_{5}(t), T_{6}(t)$ and $T_{7}(t)$ are classified as below,

$$
\begin{aligned}
\left\|T_{1} n(t)\right\|=\| & \frac{2(1-\tau)}{2-\tau M(\tau)} \Phi_{1}\left(t, S_{B} n\right)-\Phi_{1}\left(t, S_{B}(n-1)\right. \\
& +\frac{2 \tau}{2-\tau M(\tau)} \int_{0}^{t}\left(\Phi_{1}\left(y, S_{B} n\right)-\Phi_{1}\left(y, S_{B}(n-1)\right) \| d y .\right. \\
& \left\|T_{1}(t)\right\| \leq\left\|\frac{2(1-\tau)}{2-\tau M(\tau)}\right\| \Phi_{1}\left(t, S_{B} n\right)-\Phi_{1}\left(t, S_{B}(n-1) \|\right. \\
& +\frac{2 \tau}{2-\tau M(\tau)} \int_{0}^{t} \|\left(\Phi_{1}\left(y, S_{B} n\right)-\Phi_{1}\left(y, S_{B}(n-1)\right) \| d y .\right. \\
\leq & \frac{2(1-\tau)}{(2-\tau) M(\tau)} \Gamma_{1}\left\|S_{B}-S_{B}(n-1)\right\|+\frac{2 \tau}{(2-\tau) M(\tau)} \Gamma_{1}\left\|S_{B}-S_{B}(n-1)\right\| .
\end{aligned}
$$

In recurrence manner we write as

$$
\left\|T_{1}(t)\right\| \leq\left(\left(\frac{2(1-\tau)}{2-\tau M(\tau)}+\frac{2 \tau}{2-\tau M(\tau)} t_{0}\right)^{n+1} \Gamma_{1}^{n+1} .\right.
$$

Now using limit $n \rightarrow \infty$ on Eq. (34)

$$
\left\|T_{1}(t)\right\| \rightarrow 0 .
$$


Table 1 Parameters Used In BHRP And BRP Visitors Model

\begin{tabular}{ll}
\hline Notation & Parameter description \\
$n_{B}$ & Bats Birth rate \\
$n_{H}$ & Hosts Birth rate \\
$n_{p}$ & People Birth rate \\
$m_{B}$ & Bats death rate \\
$m_{H}$ & hosts death rate \\
$1 / \omega_{B}$ & Bats incubation period \\
$1 / \omega_{H}$ & Host incubation period \\
$1 / \omega_{p}$ & People incubation period \\
$1 / \hat{\omega}_{B}$ & Latent people period \\
$1 / \gamma_{B}$ & Bats infection period \\
$1 / \gamma_{H}$ & Hosts infection period \\
$1 / \gamma_{p}$ & The symptomatic people infectious period \\
$1 / \hat{\gamma}_{p}$ & The asymptomatic people infectious period \\
$\beta_{B}$ & $I_{B}$ to $S_{B}$ transmission rate \\
$\beta_{B H}$ & $I_{B}$ to $S_{H}$ transmission rate \\
$\beta_{H}$ & $I_{H}$ to $S_{H}$ transmission rate \\
$\beta_{p}$ & $I_{p}$ to $S_{p}$ transmission rate \\
$\beta_{W}$ & $W$ to $S_{p}$ transmission rate \\
\hline & \\
\hline
\end{tabular}

The same procedure using for Eq. (32) we get,

$$
\begin{gathered}
\left\|T_{2}(t)\right\| \rightarrow 0,\left\|T_{3}(t)\right\| \rightarrow 0,\left\|T_{4}(t)\right\| \rightarrow 0, \\
\left\|T_{5}(t)\right\| \rightarrow 0,\left\|T_{6}(t)\right\| \rightarrow 0,\left\|T_{7}(t)\right\| \rightarrow 0 .
\end{gathered}
$$

To show system (3) having unique solution, we suppose that there exists another solution of system (3) are $S_{1 B}(t), S_{1 p}(t), E_{1 p}(t), I_{1 p}(t), A_{1 p}(t), R_{1 P}(t)$ and $W_{1}(t)$, such that,

$$
\begin{aligned}
S_{B}(t)-S_{1 B}(t)= & \frac{2(1-\tau)}{(2-\tau) M(\tau)} \Phi_{1}\left(t, S_{B}\right)-\Phi_{1}\left(t, S_{1 B}\right) \\
& \quad+\frac{2 \tau}{(2-\tau) M(\tau)} \int_{0}^{t} \Phi_{1}\left(y, S_{B}\right)-\Phi_{1}\left(y, S_{1 B}\right) d y .
\end{aligned}
$$

Now for Eq(34) using $\|$. \|, and applying Lipschitz condition of kernelwe,

$$
\begin{gathered}
\left\|S_{B}(t)-S_{1 B}(t)\right\|\left(1-\frac{2(1-\tau)}{(2-\tau) M(\tau)} \Gamma_{1}\right. \\
\left.-\frac{2 \tau}{(2-\tau) M(\tau)} \Gamma_{1}(t)\right) \leq 0 .
\end{gathered}
$$

Conclusion: 2 The model defined in system (4) by using the strategies of Eqs. (28) and (29), we see that the succeeding relation with continivity like in Eq. (32) the terms $\hat{T}_{1}, \hat{T}_{2}, \hat{T}_{3}$, showing same behavior of Eq. (34) with assigning new supposition of solution $\hat{S}_{1}, \hat{S}_{2}, \hat{S}_{3}$, provides us Eq. (36) of the form of Eq. (35) revealed that the spreading of Corona virus in "Wahan city" do not effected by visitors (Table 1).

Theorem 10.4 The model (3) solution will unique if

$$
\left(1-\frac{2(1-\tau)}{(2-\tau) M(\tau)} \Gamma_{1}-\frac{2 \tau}{(2-\tau) M(\tau)} \Gamma_{1} t\right)>0 .
$$




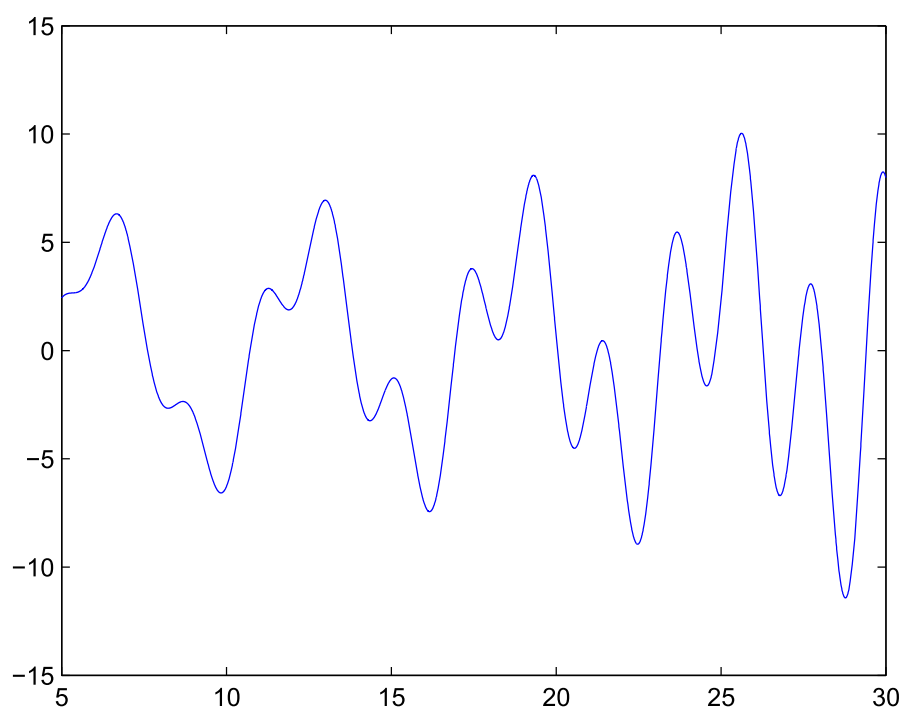

Fig. 1 The Plot Show A Random Behavior of Both population model

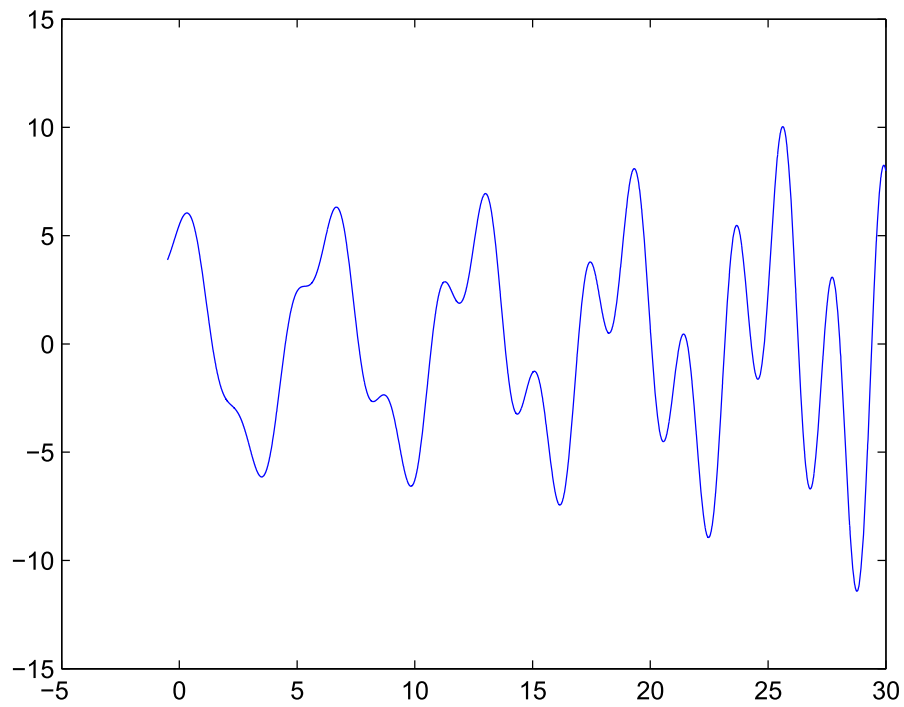

Fig. 2 The plot show a random behavior of both population model

Proof If condition defined in above theorem hold then Eq. (36) written as,

$$
\| S_{B}(t)-S_{1 B}(t 0 \|=0 .
$$

We easily get that,

$$
S_{B}(t)=S_{1 B}
$$

Provided that the following solution of all concern,

$$
S_{p}(t)=S_{1 p}(t), E_{p}(t)=E_{1 p}(t), I_{p}(t)=I_{1 p}(t),
$$




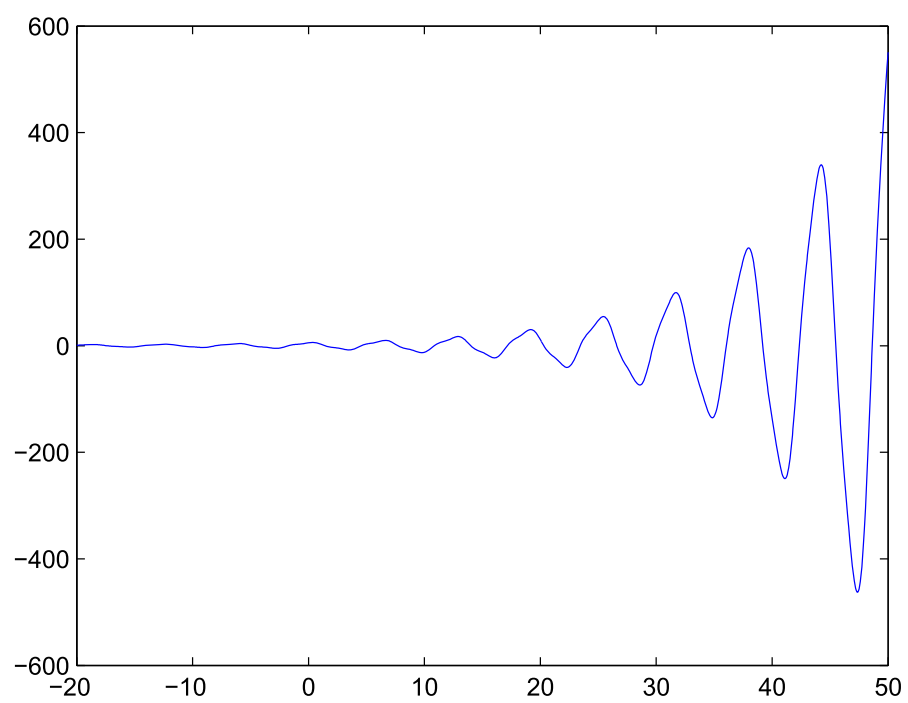

Fig. 3 The plot show a random behavior of both population model

$$
A_{p}(t)=A_{1 p}(t), R_{p}(t)=R_{1 p}(t), W(t)=W 1(t) .
$$

Similarly apply the same theorem (above) and procedure we also find the visitors population as below, provided that the following solution of all concern,

$$
S(t)=S_{1}(t), E(t)=E_{1}(t), I(t)=I_{1}(t) .
$$

\section{Conclusion}

considering the published data with calculating all parameters, we concluded that the models " $B$ H R P", and " $R P$ " showed that the spread of Corona virus is very high then MERS in any population. But the addition of our model to published data model showed that the susceptible Bats and visitors to Wuhan or any country having same estimation as that population, more specially visitors of any country are not responsible in the spread of more infection in that area. In simulation from Fig. 1 show that the spread is randomly occurred in your model which shows that, in spread of Corona virus no external agent is involved (visitors). Fig 2 also indicate that the virus may be started from any point where there will be no visitors and susceptible Bats exist. From Fig. 3 we see that the spread of this virus is very fast and then it change in any region of the country. But here our objective of this mathematical model is to estimate the role of susceptible Bats and visitors in spread of "Corona virus" in any population. Finally we say that the spread of virus is free from any type of visitors and susceptible Bats.

Acknowledgements All authors read and approved the final version.

\section{Authors' information}

MT: The corresponding author of this research article, with area of specialization are Mathematical Biology, and fluid dynamics. He is an Lecturer in Northern university, Wattar, Nowshera, Pakistan.

GZ: currently working as a Vice Chancellor at University of Malakand, lower Dir, 18800, Chakdara, Pakistan. His Ph.D from, Pusan National University, South Korea in 2008. 
SIAS: currently working a professor and dean in mathematics at Islamia College Peshawar, 25000. His Ph.D degree from Saga University Japan in 2002.

Author contributions All authors equally contributed this paper.

\section{Compliance with ethical standards}

Conflicts of interest There is no conflict of interest regarding this paper.

Open Access This article is licensed under a Creative Commons Attribution 4.0 International License, which permits use, sharing, adaptation, distribution and reproduction in any medium or format, as long as you give appropriate credit to the original author(s) and the source, provide a link to the Creative Commons licence, and indicate if changes were made. The images or other third party material in this article are included in the article's Creative Commons licence, unless indicated otherwise in a credit line to the material. If material is not included in the article's Creative Commons licence and your intended use is not permitted by statutory regulation or exceeds the permitted use, you will need to obtain permission directly from the copyright holder. To view a copy of this licence, visit http://creativecommons.org/licenses/by/4.0/.

\section{References}

1. Akgül, E.K., Akgül, A., Baleanu, D.: Laplace transform method for economic models with constant proportional caputo derivative. Fractal Fract. 4(3), 30 (2020). https://doi.org/10.3390/fractalfract4030030

2. Al-Ghafri, S., Rezazadeh, H.: Solitons and other solutions of $(3+1)$-dimensional space-time fractional modified KdV-Zakharov-Kuznetsov equation. Appl. Math. Nonlinear Sci. 4(2), 289-304 (2019)

3. Baskonus, H.M.: Complex surfaces to the fractional $(2+1)$-dimensional Boussinesq dynamical model with the local M-derivative. Eur. Phys. J. Plus 134, 322 (2019). https://doi.org/10.1140/epjp/i2019-126804

4. Bonzani, L., Mussone, L.: On the derivation of the velocity and fundamental traffic flow diagram from the modelling of the vehicle-driver behaviors. Math. Comput. Model. 50(7-8), 1107-1112 (2009)

5. Brzeziński, D.W.: Review of numerical methods for NumILPT with computational accuracy assessment for fractional calculus. Appl. Math. Nonlinear Sci. 3(2), 487-502 (2018)

6. Caputo, M., Fabrizio, M.: A new definition of fractional derivative without singular kernel. Prog. Fract. Differ. Appl. 1(2), 1-13 (2015)

7. Chen, T., Rui, J., Wang, Q., Zhao, Z., Cui, J-A., Yin, L.: A mathematical model for simulating the transmission of Wuhan novel Coronavirus. bioRxiv. 2020: 2020.2001.2019.911669. Accessed 13 Feb (2020)

8. Chen, T., Rui, J., Wang, Q., Zhao, Z., Cui, J-A., Yin, L.: A mathematical model for simulating the transmission of Wuhan novel Coronavirus. bioRxiv. 2020: 2020.2001.2019.911669. Accessed 13 Feb 2020. bibitem31A Dynamic Compartmental Mathematical Model Describing The Transmissibility Of MERS-CoV Virus In Public,Punjab University Journal of Mathematics (ISSN 1016-2526) Vol. 51(4) pp. 57-71(2019)

9. Chen, T.-M., Rui, J., Wang, Q.-P., Zhao, Z.-Y., Cui, J.-A., Yin, L.: A mathematical model for simulating the phase-based transmissibility of a novel coronavirus. Infect. Dis. Poverty, https://doi.org/10.1186/ s40249-020-00640-3

10. Chen, T., Ka-Kit Leung, R., Liu, R., Chen, F., Zhang, X., Zhao, J., et al.: Risk of imported Ebola virus disease in China. Travel Med. Infect. Dis. 12, 650-8 (2014)

11. Chen, T.M., Chen, Q.P., Liu, R.C., Szot, A., Chen, S.L., Zhao, J., et al.: The transmissibility estimation of influenza with early stage data of small-scale outbreaks in Changsha, China, 2005-2013. Epidemiol. Infect. 145, 424-33 (2017)

12. Cui, J.-A., Zhao, S., Guo, S., Bai, Y., Wang, X., Chen, T.: Global dynamics of an epidemiological model with acute and chronic HCV infections. Appl. Math. Lett. 103, 106203 (2020)

13. De la Sen, M., Agarwa, Ravi P., Ibeas, A., Alonso-Quesada, S.: On the Existence of Equilibrium Points, Boundedness, Oscillating Behavior and Positivity of a SVEIRS Epidemic Model under Constant and Impulsive Vaccination,Advances in Difference Equations Volume 2011, Article ID 748608, 32 pages https://doi.org/10.1155/2011/748608.

14. Fanelli, D., Piazza, F.: Analysis and forecast of COVID-19 spreading in China. Italy and France. Chaos Solitons Fractals 134, 109761 (2020). https://doi.org/10.1016/j.chaos.2020.109761 
15. Gao, W., Baskonus, H.M., Shi, L.: New investigation of bats-hosts-reservoir-people coronavirus model and application to 2019-nCoV system. Adv Differ Equ 2020, 391 (2020)

16. Gao, W., Senel, M., Yel, G., Baskonus, H.M., Senel, B.: New complex wave patterns to the electrical transmission line model arising in network system. AIMS Math. 5(3), 1881-1892 (2020). https://doi.org/ 10.3934/math.2020125

17. Gao, W., Veeresha, P., Prakasha, D.G., Baskonus, H.M., Yel, G.: New numerical results for the timefractional Phi-four equation using a novel analytical approach. Symmetry. 12(3), 478 (2020). https://doi. org/10.3390/sym12030478

18. Gao, W., Yel, G., Baskonus, H.M., Cattani, C.: Complex solitons in the conformable (2+1)-dimensional Ablowitz-Kaup-Newell-Segur equation. AIMS Math. 5(1), 507-521 (2019). https://doi.org/10.3934/ math.2020034

19. Kumar, S., Nisar, K.S., Kumar, R., Cattani, C., Semet, B.: A new Rabotnov fractional-exponential functionbased fractional derivative for diffusion equation under external force. Math. Methods Appl. Sci. (2020). https://doi.org/10.1002/mma.6208

20. Kumar, D., Singh, J., Al Qurashi, M., Baleanu, D.: A new fractional SIRS-SI malaria disease model with application of vaccines, antimalarial drugs, and spraying. Adv. Differ. Equ. 2019, 278 (2019)

21. Li, Q., Guan, X., Wu, P., Wang, X., Zhou, L., Tong, Y., et al.: Early transmission dynamics in Wuhan, China, of novel coronavirus-infected pneumonia. N Engl. J. Med. (2020). https://doi.org/10.1056/ NEJMoa2001316

22. Liu, J., Zhang, T.: Global stability for a tuberculosis model. Math. Comput. Model 54, 836-845 (2011)

23. Longini Jr., I.M., Nizam, A., Xu, S., Ungchusak, K., Hanshaoworakul, W., Cummings, D.A., et al.: Containing pandemic influenza at the source. Science. 309, 1083-7 (2005)

24. Losada, J., Nieto, J.J.: Properties of a new fractional derivative without singular kernel. Prog. Fract. Differ. Appl. 1(2), 87-92 (2015)

25. Pand, S.K., Abdeljawad, T., Ravichandran, C.: A complex valued approach to the solutions of RiemannLiouville integral, Atangana-Baleanu integral operator and non-linear telegraph equation via fixed point method. Chaos Solitons Fractals. 130, 109439 (2020)

26. Rushchitsky, J.J., Simchuk, Y.V.: Modeling cylindrical waves in nonlinear elastic composites. Int. Appl. Mech. 43, 638-646 (2007)

27. Singh, J., Kumar, D., Hammouch, Z., Atangana, A.: A fractional epidemiological model for computer viruses pertaining to a new fractional derivative. Appl. Math. Comput. 316, 504-515 (2018). https://doi. org/10.1016/j.amc.2017.08.048

28. Singh, J., Kumar, D., Baleanu, D.: On the analysis of fractional diabetes model with exponential law. Adv. Differ. Equ. 2018(1), 231 (2018)

29. Tahir, et al.: Ebola virus epidemic disease its modeling and stability analysis required abstain strategies. Cogent Biol. 4, 1488511 (2018). https://doi.org/10.1080/23312025.2018.1488511

30. Tahir M., et al.: Stability Behaviour of Mathematical Model MERS Corona Virus Spread in Population. Filomat 33:12 , 3947-3960 (2019) https://doi.org/10.2298/FIL1912947T

31. Tahir, M., Nousheen, A., syed I., Ali S., Tahir K.: Modeling and stability analysis of epidemic expansion disease Ebola virus with implications prevention in population, Cogent Biology 5, 1619219(2019). https:// doi.org/10.1080/23312025.2019.1619219

32. Tahir, M., Shah, S., Inayat A., Zaman, G., Khan, T.: A Dynamic Compartmental Mathematical Model Describing The Transmissibility Of MERS-CoV Virus In Public,Punjab University Journal of Mathematics (ISSN 1016-2526) Vol. 51(4)(2019) pp. 57-71

33. Tahir, M., Shah, S.I.A., Zaman, G.: Prevention strategy for superinfection mathematical model tuberculosis and HIV associated with AIDS. Cogent Math. Stat. 6, 1637166 (2019). https://doi.org/10.1080/ 25742558.2019.1637166

34. World Health Organization. Coronavirus. World Health Organization, cited January 19, 2020. Available: https://www.who.int/health-topics/coronavirus

35. Yokuş, A., Gülbahar, S.: Numerical solutions with linearization techniques of the fractional Harry Dym equation. Appl. Math. Nonlinear Sci. 4(1), 35-42 (2019)

36. Zhang, S., Hu, Q., Deng, Z., Hu, S., Liu, F., Yu, S., et al.: Transmissibility of acute haemorrhagic conjunctivitis in small-scale outbreaks in Hunan Province, China. Sci. Rep. 10, 119 (2020)

Publisher's Note Springer Nature remains neutral with regard to jurisdictional claims in published maps and institutional affiliations. 